\title{
The Curved N-Body Problem: Risks and Rewards
}

\author{
FLoRIN Diacu
}

写 xploring new mathematical territory can be a risky 7 adventure. With no landmarks in sight, it's easy to

err, as all the giants, from Fermat and Newton to Gauss and Poincaré, well knew. Still, an expedition into the unknown is highly rewarding if planned with care and advanced with restraint, a thing the masters knew too. I learned this lesson not only by reading them and about them, but also from experience.

\section{A Brief History of the Problem}

The curved $N$-body problem is a short name for the equations that describe the motion of gravitating particle systems in spaces of constant Gaussian curvature. I discovered this universe a few years ago, and found it fascinating. Later I also looked into its history, which proved to be as gripping as the universe itself.

The classical $N$-body problem has its roots in the work of Isaac Newton, who introduced it in his masterpiece Principia in 1687. His goal was to study the motion of the moon, and for this he offered a model for the dynamics of celestial objects under gravitational attraction. It took about 150 years until the independent founders of hyperbolic geometry, János Bolyai and Nikolai Lobachevsky, thought of extending gravitation to spaces of constant curvature [4, 28].

By that time, Gauss had already derived his gravity law, which is equivalent to Newton's, but emphasizes that the gravitational force is inversely proportional to the area of the sphere of radius equal to the distance between the attracting bodies. So it's no wonder that both Bolyai and Lobachevsky saw the connection between the geometry of the space and the laws of physics. They defined a Kepler problem in the hyperbolic space $\mathbb{H}^{3}$ (the gravitational motion of a body about a fixed point) in which the force is inversely proportional to the area of a sphere of radius equal to the hyperbolic arc distance between the body and the attraction centre. Alas, they never followed this idea to obtain the equations of motion in analytic form [4, 28].

One mathematician who appreciated the problem was Lejeune Dirichlet. He told friends he had dealt with it during his last year in Berlin (1852), but he never published anything in this direction [27]. It was Ernest Schering, a Göttingen mathematician and reluctant editor of Gauss's posthumous work, who came up with an analytic expression of the potential in 1870 [33]. Since the area of a sphere in $\mathbb{H}^{3}$ is proportional to $\sinh ^{2} r$, where $r$ is its radius, Schering proposed a force, $F$, inversely proportional to this function, which led him to a potential, $U$, that involves coth $r$, because $F=\nabla U$. But was this the right way to extend Newton's law? After all, there are many ways to do that. Rudolf Lipschitz must have asked this question, for in 1873 he defined a potential in $\mathbb{S}^{3}$ proportional to $1 / \sin r$. His attempts to solve the Kepler problem led him to expressions that depend on elliptic integrals, which cannot be explicitly solved, so his approach was a dead end. In 1885, Wilhelm Killing returned to the original idea and used $\cot r$ for the potential in $\mathbb{S}^{3}$ [22]. Still, without knowing whether the orbits following this gravitational law are conic sections, as it happens in the Euclidean case, a question mark loomed over this extension of gravity to spaces of constant curvature.

The breakthrough came at the dawn of the 20th century thanks to Heinrich Liebmann, a mathematician known today mostly for his work in hyperbolic geometry. In 1902, Liebmann published a paper in which he showed that the orbits of the curved Kepler problem are indeed conic 
sections in the hyperbolic plane and that the coth potential is a harmonic function in $3 \mathrm{D}$, but not in $2 \mathrm{D}$, the same as in the classical Kepler problem [25]. His next key result was related to Bertrand's theorem, which states that for the Kepler problem there are only two analytic central potentials in the Euclidean space for which all bounded orbits are closed, one of the potentials being the inverse square law [2]. In 1903, Liebmann proved a similar theorem for the cotangent potential in $\mathbb{H}^{2}$ and $\mathbb{S}^{2}$ [26]. These properties established the law proposed by Bolyai and Lobachevsky as the natural extension of gravity to spaces of constant curvature, and explained why the inverse square of the arc distance, which seems more natural but doesn't have these features, is not the way to go.

This great news, which meant that my research on the curved $\mathrm{N}$-body problem was on the right track, also made me curious about Liebmann. I learned that he had been briefly the rector of the University of Heidelberg, my own doctoral Alma Mater, but had been ousted from his post soon after the Nazis came to power, during their abominable purge of Jewish intellectuals. In 2009, I visited Heidelberg again, and learned from my Ph.D. supervisor, Willi Jäger, about the Liebmann Remembrance Colloquium of 2008. Liebmann's son Karl-Otto, also an academic, had donated on this occasion an oil portrait of his father to the university. This painting has now a place of honour in the Faculty of Mathematics.

Not much was done after Liebmann's breakthrough. Before the recent publication of a paper by the Spanish mathematicians Cariñena, Rañada, and Santander [3], which

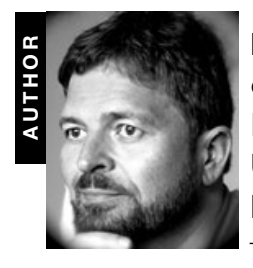

FLORIN DIACU obtained his mathematics diploma from the University of Bucharest in Romania and his Ph.D. degree from the University of Heidelberg in Germany. He has been teaching at the University of Victoria in Canada for more than two decades. Apart from mathematics research, he enjoys writing nonfiction and poetry. His latest book, Megadisasters - The Science of Predicting the Next Catastrophe, published by Princeton University Press in North America and Oxford University Press in the rest of the English-speaking world, won a "Best Academic Book Award" in 20 I I. In his leisure time he enjoys reading, playing tennis, and hiking.

Pacific Institute for the Mathematical Sciences and

Department of Mathematics and Statistics University of Victoria

Victoria, BC

Canada V8W 3R4

e-mail: diacu@math.uvic.ca had been my source of inspiration, the only important development was the work on the 2-body problem initiated by the Russian school of celestial mechanics, led by Valery Kozlov [23]. These researchers discovered that, unlike in Euclidean space, where the Kepler problem and the 2-body problem are equivalent, in curved space the equations of motion are distinct. Moreover, the curved Kepler problem is integrable, whereas the curved 2-body problem is not [34].

When I wanted to extend the curved 2-body problem to any number $N \geq 2$ of bodies, the only reference point I had was the paper of the Spanish mathematicians. I learned about the work of Schering, Killing, and Liebmann only months after discovering this new universe. But before getting into mathematical details, I will recount how everything started.

\section{Beginnings}

We feel restless without a good problem to solve, a point in life we can reach for various reasons, including loss of interest in what we've been doing. Most of us hit this wall sooner or later, and so did I. Finding a new appealing subject can be challenging under such circumstances. But in this case chance favoured me.

I had worked in the dynamics of particle systems for more than 20 years. I started with the classical $N$-body problem and later introduced the Manev model and its generalization, the quasihomogeneous law, which includes those of Newton, Birkhoff, Coulomb, Schwarzschild, Lennard-Jones, Van der Waals, and a few others. The mathematical community embraced the topic and produced some 200 papers so far. But a few years ago I felt tired of studying the same equations and ready for a new challenge.

I was teaching a course in non-Euclidean geometry, focusing on the hyperbolic plane and using synthetic methods and complex functions in the Poincaré and KleinBeltrami disks and the Poincaré upper-half plane. I also used Weierstrass's model (often wrongly attributed to Lorentz), the natural analogue to the sphere $\mathbb{S}^{2}$ of elliptic geometry. To define it, take the upper sheet of the

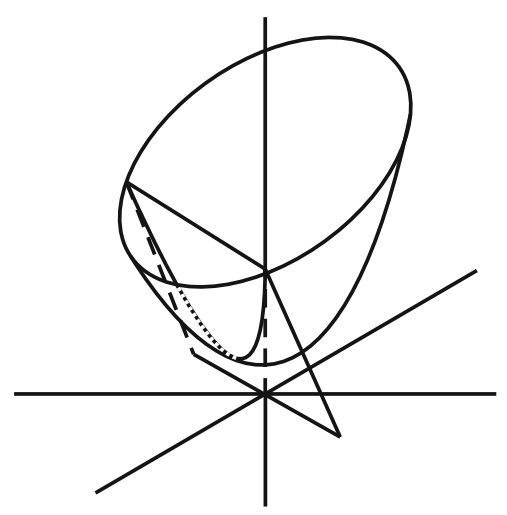

Figure I. Weierstrass's model of the hyperbolic plane (given by the upper sheet of the hyperboloid of two sheets) is equivalent to the Poincaré disk model, a fact that can be proved by stereographic projection. 
hyperboloid of two sheets, $\mathbb{H}^{2}=\left\{(x, y, z) \mid x^{2}+y^{2}-z^{2}=\right.$ $-1, z>0\}$ (see Figure 1). The geodesics are the hyperbolas obtained by intersecting this surface with the planes that contain the frame's origin. Through a point outside a geodesic, we can draw two parallel geodesics that meet the original one at infinity, each at a different end, so the main axiom of hyperbolic geometry is satisfied.

Indeed, take a geodesic $\gamma$, i.e., a hyperbola obtained by intersecting a plane through the origin, $O$, of the coordinate system with $\mathbb{H}^{2}$. This hyperbola has two asymptotes in its plane: the straight lines $a$ and $b$, intersecting at $O$. Take a point, $P$, on the surface that is not on the chosen hyperbola. The plane $a P$ produces the geodesic hyperbola $\alpha$, whereas $b P$ produces $\beta$. These two hyperbolas intersect at $P$. Then $\alpha$ and $\gamma$ meet at infinity along $a$, whereas $\beta$ and $\gamma$ meet at infinity along $b$. All the hyperbolas between $\alpha$ and $\beta$ (also obtained from planes through $O$ ) are non-secant with $\gamma$.

But unlike $\mathbb{S}^{2}$, which is embedded in $\mathbb{R}^{3}, \mathbb{H}^{2}$ is embedded in the 3D Minkowski space, i.e., $\mathbb{R}^{3}$ with the Lorentz inner product, which has the $(+,+,-)$ signature instead of the standard $(+,+,+)$. A different inner product affects geometric orthogonality. Indeed, a tangent vector to $\mathbb{S}^{2}$ and the radius vector touching it are orthogonal, i.e., their inner product is zero. In $\mathbb{H}^{2}$ things change. The vector from the origin and the corresponding tangent vector don't look orthogonal, but their Lorentz inner product is zero, so they are perpendicular to each other in the Minkowski space. Moreover, this inner product is responsible for the negative curvature of $\mathbb{H}^{2}$, which appears positively curved to the naked eye.

I also happened to read at that time the paper about the Kepler problem in $\mathbb{S}^{2}$ and $\mathbb{H}^{2}$ by Cariñena, Rañada, and Santander [3]. The force between the two points acts along the geodesic that connects them; in $\mathbb{S}^{2}$, the direction is that of the shortest distance, except for the antipodal case, when a singularity occurs. The potential defining the equations uses the cotangent of the arc distance in $\mathbb{S}^{2}$ and the cotangent hyperbolic of the arc distance in $\mathbb{H}^{2}$. As I explained earlier, this potential, and not the inverse arc distance-as intuition suggests - is the natural extension of the Newtonian model to curved space.

The Spanish mathematicians had written the equations of motion in intrinsic coordinates, using differential geometry, and obtained conic sections as orbits in $\mathbb{S}^{2}$ and $\mathbb{H}^{2}$, in agreement with what we know from $\mathbb{R}^{2}$. This result gave me the idea to find the equations of the curved $N$ body problem, so I tried the intrinsic approach. But the computations proved complicated, so I emailed my friends Manuele Santoprete, a former student of mine, now a professor at Wilfrid Laurier University, and my old collaborator Ernesto Pérez-Chavela of Mexico City, hoping that we could find a new approach. They eagerly accepted my challenge. We exchanged emails for weeks, during which a lucky combination of ideas shaped up. The insight we achieved in this way proved crucial. In the next two sections I will summarize what we found during the couple of months that followed. Later, after obtaining the equations of motion, thus knowing what to expect, we also derived them in intrinsic coordinates $[16,31]$.

\section{Deriving the Equations of Motion}

In $2 \mathrm{D}$, the right setting for the problem is on spheres and hyperbolic spheres, i.e.,

$$
\begin{aligned}
& \mathbb{S}_{\kappa}^{2}=\left\{(x, y, z) \mid x^{2}+y^{2}+z^{2}=\kappa^{-1}\right\} \text { and } \\
& \mathbb{H}_{\kappa}^{2}=\left\{(x, y, z) \mid x^{2}+y^{2}-z^{2}=\kappa^{-1}, z>0\right\}
\end{aligned}
$$

respectively, depending on the sign of the curvature $\kappa$. When $\kappa \rightarrow 0$ we recover $\mathbb{R}^{2}$ (see Figure 2 ). These manifolds are embedded in the ambient space, which is $\mathbb{R}^{3}$ with the standard inner product, for positive curvature, but $\mathbb{R}^{3}$ with the Lorentz inner product, i.e., the Minkowski space $\mathbb{R}^{2,1}$, for negative curvature. In other words, the inner product of the vectors $\mathbf{a}=\left(a_{x}, a_{y}, a_{z}\right)$ and $\mathbf{b}=\left(b_{x}, b_{y}, b_{z}\right)$ is $\mathbf{a} \cdot \mathbf{b}=a_{x} b_{x}+a_{y} b_{y}+\sigma a_{z} b_{z}$, where $\sigma=1$ for $\kappa>0$ and $\sigma=-1$ for $\kappa<0$.

The Lagrangian formulation of mechanics can be obtained from a basic variational principle that minimizes a function called action,

$$
A=\int_{t_{1}}^{t_{2}} L d t
$$

where $L=T-V$ is the Lagrangian function, with $T$ and $V$ denoting the kinetic and the potential energy of a certain mechanical system and $t_{1}<t_{2}$ are two instants in time. Such a minimum leads to the differential equations that describe the motion.

The theory of constrained Lagrangian dynamics adds some conditions, which in our case are those of keeping the bodies $m_{1}, m_{2}, \ldots, m_{N}$ on the manifold [20]. For our problem, if $\mathbf{q}_{i}=\left(x_{i}, y_{i}, z_{i}\right)$ are the position vectors, $\dot{\mathbf{q}}_{i}=\left(\dot{x}_{i}, \dot{y}_{i}, \dot{z}_{i}\right)$ the velocities, and the constraints are characterized by the equations $f_{i}=0, i=1,2, \ldots, N$, then the motion is described by the Euler-Lagrange equations with constraints,

$$
\frac{d}{d t}\left(\frac{\partial L}{\partial \dot{\mathbf{q}}_{i}}\right)-\frac{\partial L}{\partial \mathbf{q}_{i}}-\lambda_{i}(t) \frac{\partial f_{i}}{\partial \mathbf{q}_{i}}=\mathbf{0}, \quad i=1,2, \ldots, N,
$$

where the functions $\lambda_{i}, i=1,2, \ldots, N$, are the Lagrange multipliers.

To bring the positive and negative curvature cases into one equation, we can follow the Spanish mathematicians and unify circular and hyperbolic trigonometry. For this, we define the $\kappa$-sine as

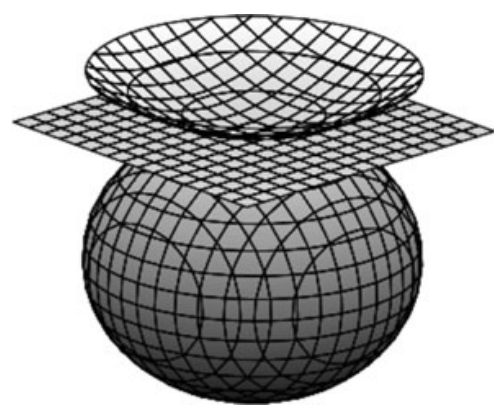

Figure 2. The continuous deformation of the sphere into a plane into a hyperbolic sphere. 


$$
\operatorname{sn}_{\kappa}(x):= \begin{cases}\kappa^{-1 / 2} \sin \left(\kappa^{1 / 2} x\right) & \text { if } \kappa>0 \\ x & \text { if } \kappa=0 \\ (-\kappa)^{-1 / 2} \sinh \left((-\kappa)^{1 / 2} x\right) & \text { if } \kappa<0,\end{cases}
$$

the $\kappa$-cosine as

$$
\operatorname{csn}_{\kappa}(x):= \begin{cases}\cos \left(\kappa^{1 / 2} x\right) & \text { if } \kappa>0 \\ 1 & \text { if } \kappa=0 \\ \cosh \left((-\kappa)^{1 / 2} x\right) & \text { if } \kappa<0\end{cases}
$$

as well as the $\kappa$-tangent and $\kappa$-cotangent as

$$
\operatorname{tn}_{\kappa}(x):=\frac{\operatorname{sn}_{\kappa}(x)}{\operatorname{csn}_{\kappa}(x)} \quad \text { and } \quad \operatorname{ctn}_{\kappa}(x):=\frac{\operatorname{csn}_{\kappa}(x)}{\operatorname{sn}_{\kappa}(x)},
$$

respectively. The fundamental formula of unified trigonometry becomes

$$
\kappa \operatorname{sn}_{\kappa}^{2}(x)+\operatorname{csn}_{\kappa}^{2}(x)=1 .
$$

For fun, students can try to derive other formulas.

The distance between two points $\mathbf{a}, \mathbf{b} \in \mathbb{R}^{3}$ can be defined as

$$
d_{\kappa}(\mathbf{a}, \mathbf{b}):=(\sigma \kappa)^{-1 / 2} \operatorname{csn}_{\kappa}^{-1}\left(\frac{\kappa \mathbf{a} \cdot \mathbf{b}}{\sqrt{\kappa \mathbf{a} \cdot \mathbf{a}} \sqrt{\kappa \mathbf{b} \cdot \mathbf{b}}}\right),
$$

where $\operatorname{csn}_{\kappa}^{-1}$ is the inverse trigonometric function of $\operatorname{csn}_{\kappa}$. On $\mathbb{S}_{\kappa}^{2}$ and $\mathbb{H}_{\kappa}^{2}$ this is the arc distance. For $\kappa \rightarrow 0$, we recover the Euclidean norm.

If we further define the kinetic energy $T$ as

$$
T_{\kappa}(\mathbf{q}, \dot{\mathbf{q}}):=\frac{1}{2} \sum_{i=1}^{N} m_{i}\left(\dot{\mathbf{q}}_{i} \cdot \dot{\mathbf{q}}_{i}\right)\left(\kappa \mathbf{q}_{i} \cdot \mathbf{q}_{i}\right),
$$

the potential energy as $V=-U_{\kappa}$, where

$$
U_{\kappa}(\mathbf{q}):=\sum_{1 \leq i<j \leq N} m_{i} m_{j} \operatorname{ctn}_{\kappa}\left(d_{\kappa}\left(\mathbf{q}_{i}, \mathbf{q}_{j}\right)\right)
$$

is the force function, and the constraints with the help of the functions $f_{i}=x_{i}^{2}+y_{i}^{2}+\sigma z_{i}^{2}-\kappa^{-1}, i=1,2, \ldots, N$, the equations of motion take the form

$$
m_{i} \ddot{\mathbf{q}}_{i}=\nabla_{\mathbf{q}_{i}} U_{\kappa}(\mathbf{q})-\kappa m_{i}\left(\dot{\mathbf{q}}_{i} \cdot \dot{\mathbf{q}}_{i}\right) \mathbf{q}_{i}, \quad i=1,2, \ldots, N,
$$

where $\nabla_{\mathbf{q}_{i}}:=\left(\partial_{x_{i}}, \partial_{y_{i}}, \sigma \partial_{z_{i}}\right)$ is the gradient operator.

When $\kappa \rightarrow 0$, the velocity terms obviously vanish, the arc distance tends to the Euclidean one, and the potential becomes the standard Newtonian potential, in other words we recover the classical equations of motion, which we have thus extended to spaces of constant curvature. The force acts along geodesics: if two bodies are placed on the manifold with zero initial velocities, they will move along the common geodesic until they collide, the same as in the Euclidean case. So everything makes sense from the physical point of view.

The next things to look for are the first integrals, since they not only allow the reduction of the system, but also have useful physical meaning.

\section{First Integrals}

Before finding the first integrals, let's simplify the problem. With the coordinate- and time-rescaling transformations

$$
\mathbf{q}_{i}=|\kappa|^{-1 / 2} \mathbf{r}_{i}, \quad i=1,2, \ldots, N, \text { and } \tau=|\kappa|^{3 / 4} t,
$$

and redenoting the new position vectors $\mathbf{r}_{i}$ by $\mathbf{q}_{i}$ and the fictitious time $\tau$ by $t$, the equations of motion can be written as:

$$
\begin{array}{r}
\ddot{\mathbf{q}}_{i}=\sum_{j=1, j \neq i}^{N} \frac{m_{j}\left[\mathbf{q}_{j}-\sigma\left(\mathbf{q}_{i} \cdot \mathbf{q}_{j}\right) \mathbf{q}_{j}\right]}{\left[\sigma-\sigma\left(\mathbf{q}_{i} \cdot \mathbf{q}_{j}\right)^{2}\right]^{3 / 2}}-\sigma\left(\dot{\mathbf{q}}_{i} \cdot \dot{\mathbf{q}}_{i}\right) \mathbf{q}_{i}, \quad \mathbf{q}_{i} \cdot \mathbf{q}_{i}=\sigma, \\
i=1,2, \ldots, N .
\end{array}
$$

So for positive curvature, the problem is reduced to $\mathbb{S}^{2}$, and for negative curvature to $\mathbb{H}^{2}$. It is not difficult to see that the equations of motion are Hamiltonian, with the energy integral given by

$$
T(\mathbf{q}, \dot{\mathbf{q}})-U(\mathbf{q})=h,
$$

where $b$ is the energy constant and

$$
\begin{aligned}
& T(\mathbf{q}, \dot{\mathbf{q}})=\frac{1}{2} \sum_{i=1}^{N} m_{i}\left(\dot{\mathbf{q}}_{i} \cdot \dot{\mathbf{q}}_{i}\right)\left(\sigma \mathbf{q}_{i} \cdot \mathbf{q}_{i}\right), \\
& U(\mathbf{q})=\sum_{j=1, j \neq i}^{N} \frac{\sigma m_{i} m_{j} \mathbf{q}_{i} \cdot \mathbf{q}_{j}}{\left[\sigma\left(\mathbf{q}_{i} \cdot \mathbf{q}_{i}\right)\left(\mathbf{q}_{j} \cdot \mathbf{q}_{j}\right)-\sigma\left(\mathbf{q}_{i} \cdot \mathbf{q}_{j}\right)^{2}\right]^{1 / 2}}
\end{aligned}
$$

are the kinetic energy and force function, respectively, in the new coordinates. The total angular momentum produces three more integrals:

$$
\sum_{i=1}^{N} m_{i} \mathbf{q}_{i} \times \dot{\mathbf{q}}_{i}=\mathbf{c},
$$

where $\mathbf{c}$ is a constant 3 -vector and $x$ denotes the cross product.

The equations of motion generalize easily to any higher dimension, but the ones of physical interest so far are to $\mathbb{S}^{3}$ and $\mathbb{H}^{3}$. For this, we introduce the coordinates $(w, x, y, z)$ in $\mathbb{R}^{4}$. With this in mind, the equations of motion and the energy integral have the same form as above. But the angular momentum integrals change since the concept of cross product has no meaning in $\mathbb{R}^{4}$. We need to employ the exterior wedge product, $\wedge$, which leads to 6 angular momentum integrals:

$$
\sum_{i=1}^{N} m_{i} \mathbf{q}_{i} \wedge \dot{\mathbf{q}}_{i}=\mathbf{C}
$$

where $\mathbf{C}=c_{w x} \mathbf{e}_{w} \wedge \mathbf{e}_{x}+c_{w y} \mathbf{e}_{w} \wedge \mathbf{e}_{y}+c_{w z} \mathbf{e}_{w} \wedge \mathbf{e}_{z}+c_{x y} \mathbf{e}_{x} \wedge \mathbf{e}_{y}+$ $c_{x z} \mathbf{e}_{x} \wedge \mathbf{e}_{z}+c_{y z} \mathbf{e}_{y} \wedge \mathbf{e}_{z}$, with $\mathbf{e}_{w}=(1,0,0,0), \mathbf{e}_{x}=(0,1,0,0)$, $\mathbf{e}_{y}=(0,0,1,0)$, and $\mathbf{e}_{z}=(0,0,0,1)$ being the unit vectors in $\mathbb{R}^{4}$, whereas their wedge products form a basis of bivectors in the corresponding exterior algebra. The real coefficients $c_{w x}$, $c_{w y}, c_{w z}, c_{x y}, c_{x z}, c_{y z}$ express the rotation of the system relative to the planes given by their indices. Indeed, although it makes sense in $\mathbb{R}^{3}$ to define rotation relative to an axis, it is better to think of rotations in $\mathbb{R}^{4}$ relative to an invariant (but not pointwise fixed) plane.

Unlike in Euclidean space, there are no integrals of the centre of mass and linear momentum. This fact is not unexpected, given that these first integrals don't exist for $\mathrm{N}$ body problems obtained by discretizing Einstein's field 
equations, as done by Levi-Civita [24], Einstein, Infeld, and Hoffmann [18], or Fock [19]. So, in general, there are no points like the centre of mass of a particle system in the Euclidean case, where forces cancel each other or make the centre move uniformly along a geodesic. Therefore the dimension of the phase space $(\mathbf{q}, \dot{\mathbf{q}})$ is $4 N-4$ in $\mathbb{S}^{2}$ and $\mathbb{H}^{2}$, since there are 4 first integrals, but $6 N-7$ in $\mathbb{S}^{3}$ and $\mathbb{H}^{3}$, because there are 7 first integrals.

\section{Singularities}

These equations of motion open a new world. Any mathematician would now want to find some solutions, hoping to understand how they behave. For given initial data, standard results of the theory of ordinary differential equations ensure the existence and uniqueness of an analytic solution, defined locally on some interval, say, $\left[0, t^{+}\right)$. Such a solution can be analytically extended to an interval $\left[0, t^{*}\right)$, with $0<t^{+}<t^{*}$. If $t^{*}=\infty$, the solution is globally defined, i.e., the motion of the bodies takes place forever. But can it happen that $t^{*}$ is finite?

To answer this question, we need to determine whether the equations of motion have singularities. A brief look reveals that they do. Whenever $\mathbf{q} \in \Delta$, where

$$
\Delta=\cup_{1 \leq i<j \leq N}\left\{\mathbf{q} \mid\left(\mathbf{q}_{i} \cdot \mathbf{q}_{j}\right)^{2}=1\right\},
$$

at least a denominator cancels, so the equations fail to make sense. Consequently, for a solution $\mathbf{q}$, the time $t^{*}$ could be finite if $\lim _{t \rightarrow t^{*}} \mathbf{q}(t) \in \Delta$. To understand what happens, notice first that $\Delta=\Delta^{+} \cup \Delta^{-}$, where

$$
\begin{aligned}
& \Delta^{+}=\cup_{1 \leq i<j \leq N}\left\{\mathbf{q} \mid \mathbf{q}_{i} \cdot \mathbf{q}_{j}=1\right\} \text { and } \\
& \Delta^{-}=\cup_{1 \leq i<j \leq N}\left\{\mathbf{q} \mid \mathbf{q}_{i} \cdot \mathbf{q}_{j}=-1\right\} .
\end{aligned}
$$

A close look shows that, in $\mathbb{H}^{2}$ or $\mathbb{H}^{3}$, if $\mathbf{q}$ tends to an element of $\Delta$, its limit must be an element of $\Delta^{-}$, which represents a collision between at least two bodies. In $\mathbb{S}^{2}$ or $\mathbb{S}^{3}$, however, if $\mathrm{q}$ tends to an element of $\Delta$, the solution could end up in a collision configuration, if the element is in $\Delta^{+}$, an antipodal configuration, if the element is in $\Delta^{-}$, or a collision-antipodal configuration, which requires at least 3 bodies and that the element be in the union $\Delta^{+} \cup \Delta^{-}$. So while in hyperbolic space there are no surprises, spherical space reveals something new.

Further investigation shows that, for 2 bodies, collisions on the sphere are "attractive," whereas antipodal singularities are "repelling." More precisely, if 2 bodies are placed close to each other with zero initial velocities, they soon collide. But if 2 bodies are placed with zero initial velocities close to the opposite sides of a diameter, they move fast away from each other along the common geodesic, just to end up in a collision, too.

Things become even more interesting with the collisionantipodal singularities. For simplicity, let us consider an isosceles problem on the equator $z=0$, as shown in Figure 3 . The bodies $m_{1}$ and $m_{2}$, which we keep symmetric relative to the vertical axis, have equal mass $M$, whereas the body $m_{3}$, which stays fixed at $(x, y)=(0,-1)$, has mass $m$. The study of this problem teaches us that anything can happen relative to a the collision-antipodal configuration

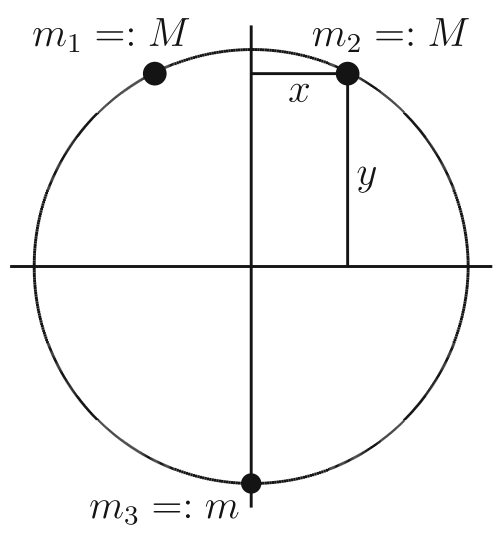

Figure 3. The initial positions of $m_{1}, m_{2}$, and $m_{3}$ on the geodesic $z=0$.

corresponding to $m_{1}$ and $m_{2}$ at $(0,1)$ and $m_{3}$ at $(0,-1)$. More precisely:

(i) for $M=8 m$, if the bodies are placed with zero initial velocities close enough to the above collision-antipodal configuration, they end up in a collision-antipodal singularity at finite $t^{*}$

(ii) for $M=2 m$, if the bodies are placed, with zero initial velocities, no matter how close to the collision-antipodal configuration, they move away from it, never to reach it;

(iii) for $M=4 m$, there exist initial positions, corresponding to zero initial velocities, for which the bodies reach the collision-antipodal configuration, but the solution remains analytic at $t^{*}$, so it does not encounter a singularity there [15].

Part of this highly unusual behaviour can be explained by the following result, which extends to $\mathbb{S}^{2}$ a theorem Painlevé proved in 1896 for the classical 3-body problem. The curved version below says that, to have expectable behaviour, we should stay away from collision-antipodal configurations [5].

If $(\mathbf{q}, \dot{\mathbf{q}})$ is an analytic solution of the curved 3-body problem in $\mathbb{S}^{2}$, defined on $\left[0, t^{*}\right)$, with $t^{*}$ a singularity, then

$$
\lim _{t \rightarrow t^{*}} \min _{1<i \leq j<n}\left|\left(\mathbf{q}_{i} \cdot \mathbf{q}_{j}\right)^{2}-1\right|=0 .
$$

Conversely, assume that $(\mathbf{q}, \dot{\mathbf{q}})$ is an analytic solution, defined on $\left[0, t^{*}\right)$, that is bounded away from collisionantipodal configurations. Then, if

$$
\lim _{t \rightarrow t^{*}} \min _{1<i \leq j<n}\left|\mathbf{q}_{i} \cdot \mathbf{q}_{j}-1\right|=0,
$$

$t^{*}$ is a singularity of the solution.

But to understand what happens from the physical point of view, we have to take a better look at the force function and its gradient.

\section{A Cosmological Detour}

Our intuition for gravitation follows the Newtonian view in Euclidean space: the larger the distance, the weaker the force. This model stays unchanged in the hyperbolic case. But things are different on the sphere. Let's start with $N=2$ 
on $\mathbb{S}^{2}$ and fix a body initially at the north pole. The potential and the norm of its gradient are, respectively,

$$
\begin{gathered}
U(\mathbf{q})=\frac{m_{1} m_{2}\left(\mathbf{q}_{1} \cdot \mathbf{q}_{2}\right)}{\left[1-\left(\mathbf{q}_{1} \cdot \mathbf{q}_{2}\right)^{2}\right]^{1 / 2}}, \\
|\nabla U(\mathbf{q})|=\left[\frac{m_{1}^{2} m_{2}^{2}\left|\mathbf{q}_{2}-\left(\mathbf{q}_{1} \cdot \mathbf{q}_{2}\right) \mathbf{q}_{1}\right|^{2}}{\left[1-\left(\mathbf{q}_{1} \cdot \mathbf{q}_{2}\right)^{2}\right]^{3}}+\frac{m_{1}^{2} m_{2}^{2}\left|\mathbf{q}_{1}-\left(\mathbf{q}_{1} \cdot \mathbf{q}_{2}\right) \mathbf{q}_{2}\right|^{2}}{\left[1-\left(\mathbf{q}_{1} \cdot \mathbf{q}_{2}\right)^{2}\right]^{3}}\right]^{1 / 2} .
\end{gathered}
$$

For various positions of the other body, $U$ ranges from $+\infty$ at collision to $-\infty$ at the antipodal configuration, being 0 when the second body is on the equator. The norm of the gradient is $+\infty$ at collision, and becomes smaller when the second body lies farther away from collision in the northern hemisphere; it takes a positive minimum value on the equator; and becomes larger the farther the second body is from the north pole while in the southern hemisphere; the norm of the gradient becomes $+\infty$ when the two bodies lie at antipodes. These remarks are also true in $\mathbb{S}^{3}$.

The qualitative behaviour described above agrees with the Newtonian gravitation in flat space only when the second body doesn't leave the northern hemisphere. But in a hypothetical spherical universe with trillions of objects ejected from a Big-Bang that took place at the north pole, all the bodies would be now still far away from the equator, since we know that, even if positive, the curvature would be extremely small. When the expanding system approaches the equator, many bodies get close to antipodal singularities, without coming close to collisions (due to the growing distance between any two bodies, as achieved by choosing the right initial conditions), so the potential energy becomes positive, like the kinetic energy. By the energy integral, the potential energy cannot grow beyond the value of the energy constant, which, when reached, makes the kinetic energy zero and stops any motion.

The larger the initial velocities, the larger the energy constant, but it is finite nevertheless, so the motion must stop at some instant, to reverse from expansion to contraction. In a highly populated spherical universe, the motion is contained in the northern hemisphere, away from the equator, never able to cross into the southern hemisphere. This happens only if all bodies are initially in the northern hemisphere, a restriction we don't have to take into consideration for a general dynamical study in $\mathbb{S}^{3}$. When the motion takes place in $\mathbb{R}^{3}$ or $\mathbb{H}^{3}$, the Big-Bang could lead to a finite, eventually collapsing, or infinite, eternally expanding universe, depending on the initial velocities taken close to a singularity.

These cosmological conclusions, which are in agreement with those of general relativity, and do not require a cosmological force, as Newtonian cosmology does in flat

Table 1. Cosmological behaviour in flat and curved classical universes

\begin{tabular}{lll}
\hline Geometry & \multicolumn{1}{c}{ Volume } & Fate \\
\hline Elliptic, $\mathbb{S}^{3}$ & Finite & Eventual collapse \\
Euclidean, $\mathbb{R}^{3}$ & Finite or infinite & Eternal expansion or eventual collapse \\
Hyperbolic, $\mathbb{H}^{3}$ & Finite or infinite & Eternal expansion or eventual collapse \\
\hline
\end{tabular}

space, are summarized in Table 1 [7]. The possibility of such applications was a welcome bonus, but I had no desire to pursue it. General relativity had already answered the cosmological questions pertaining to this model. I therefore preferred to remain focused on studying the mathematical aspects of the curved $N$-body problem.

\section{Relative Equilibria}

When entering a new world, it is always good to start from simple things and make small, but solid steps forward. In other words, it's better to understand a little than to misunderstand a lot. After taking a peek at singularities, the first problem I posed to my collaborators was that of finding relative equilibria (RE), i.e., orbits for which the system behaves like a rigid body by maintaining equal mutual distances all along the motion.

This is an active subject in the Euclidean case, where it has generated a lot of good mathematics. One of its questions made Steven Smale's list of open problems for the 21st century [35]. Saari's conjecture, still an open problem in the general case, is also related to it $[9,13,32]$. But while this is an old topic in Euclidean space, the tools used there don't work in the curved problem. Even its standard formulation is difficult to mimic in the new context, although it can be done [14].

The clue to how to start came from geometric mechanics, a research direction initiated by Ralph Abraham and Jerry Marsden, where a RE is defined as a solution generated by a one-parameter subgroup of some Lie group [1]. In this particular case, the Lie groups to look at are those that give the isometries of $\mathbb{S}^{2}, \mathbb{S}^{3}, \mathbb{H}^{2}$, and $\mathbb{H}^{3}$, i.e., $S O(3), S O(4), \operatorname{Lor}\left(\mathbb{R}^{2,1}\right)$, and $\operatorname{Lor}\left(\mathbb{R}^{3,1}\right)$, respectively. Let us focus on the second and the fourth, since the other two are respective subgroups of those. It is suitable in our case to represent them in matrix form.

After fixing a suitable basis in $\mathbb{R}^{4}$, the elements of $S O(4)$, which are orthogonal matrices of determinant 1 , have the form $P A P^{-1}$, where $P \in S O(4)$ and

$$
A=\left(\begin{array}{cccc}
\cos \theta & -\sin \theta & 0 & 0 \\
\sin \theta & \cos \theta & 0 & 0 \\
0 & 0 & \cos \phi & -\sin \phi \\
0 & 0 & \sin \phi & \cos \phi
\end{array}\right)
$$

with $\theta, \phi \in \mathbb{R}$. After fixing a suitable basis in the Minkowski space $\mathbb{R}^{3,1}$, the elements of the Lorentz group $\operatorname{Lor}\left(\mathbb{R}^{3,1}\right)$, which leave $\mathbb{H}^{3}$ invariant, are of the form $P B P^{-1}$ or $P C P^{-1}$, where $P \in \operatorname{Lor}\left(\mathbb{R}^{3,1}\right)$,

$$
\begin{aligned}
B & =\left(\begin{array}{cccc}
\cos u & -\sin u & 0 & 0 \\
\sin u & \cos u & 0 & 0 \\
0 & 0 & \cosh s & \sinh s \\
0 & 0 & \sinh s & \cosh s
\end{array}\right) \text { and } \\
C & =\left(\begin{array}{cccc}
1 & 0 & 0 & 0 \\
0 & 1 & -\xi & \xi \\
0 & \xi & 1-\xi^{2} / 2 & \xi^{2} / 2 \\
0 & \xi & -\xi^{2} / 2 & 1+\xi^{2} / 2
\end{array}\right)
\end{aligned}
$$

with $u, s, \xi \in \mathbb{R}$. 
For $A$, we call the rotations positive elliptic-elliptic if $\theta$, $\phi \neq 0$, and positive elliptic if $\theta \neq 0$ and $\phi=0$ ( or $\theta=0$ and $\phi \neq 0$, a case we ignore since it's the same as the previous); for $B$, negative elliptic if $u \neq 0$ and $s=0$, negative hyperbolic if $u=0$ and $s \neq 0$, and negative elliptic-hyperbolic if $u, s \neq 0$; for $C$, negative parabolic if $\xi \neq 0$. These possibilities lead to the natural definition of six types of RE, each corresponding to some type of rotation.

It is easy to see that the negative parabolic RE cannot satisfy all integrals of the angular momentum, so such solutions don't exist. But the other classes of RE seem to be rich, and the solutions they contain are often surprising [6, 8, 12, 14]. Among them are some orbits of the 6-body problem of equal masses in $\mathbb{S}^{3}$ for which 3 bodies are at the vertices of an equilateral triangle inscribed in the circle

$$
S_{w x}^{1}:=\left\{(w, x, y, z) \mid y^{2}+z^{2}=1, w=x=0\right\},
$$

whereas the others are at the vertices of an equilateral triangle inscribed in the circle $S_{y z}^{1}$, whose meaning is obvious from the above. For one type of solution, a triangle rotates uniformly along $S_{w x}^{1}$, whereas the other is fixed in $S_{y z}^{1}$. For the other type of solution, both triangles rotate uniformly along their respective circles, with whatever angular velocites we assign to each of them. This means that the orbits are not periodic, but quasiperiodic, a situation that cannot be encountered in $\mathbb{R}^{3}$.

How are such exotic orbits possible? The answer lies in the geometry of $\mathbb{S}^{3}$. The circles $S_{w x}^{1}$ and $S_{y z}^{1}$ are special, forming a Hopf link in a Hopf fibration, which is the mapping

$$
\begin{aligned}
\mathcal{H}: \mathbb{S}^{3} & \rightarrow \mathbb{S}^{2}, \mathcal{H}(w, x, y, z) \\
& =\left(w^{2}+x^{2}-y^{2}-z^{2}, 2(w z+x y), 2(x z-w y)\right)
\end{aligned}
$$

that takes circles of $\mathbb{S}^{3}$ to points of $\mathbb{S}^{2}$. In particular, $\mathcal{H}$ applies $S_{w x}^{1}$ to $(1,0,0)$ and $S_{y z}^{1}$ to $(-1,0,0)$. It can be proved with the help of stereographic projection that these two circles are linked like adjacent rings in a chain. Moreover, if $\mathbf{a} \in S_{w x}^{1}$ and $\mathbf{b} \in S_{y z}^{1}$, then $d(\mathbf{a}, \mathbf{b})=\pi / 2$, i.e., the arc distance between the two circles is constant. So no matter where two bodies lie (one on each of these circles), the magnitude of the attraction force between them is the same. By suitably aligning the direction of the forces acting between the two circles, as achieved with the help of two equilateral triangles, it is possible to obtain orbits like the ones described above.

Many other interesting RE exist, such as one of the 4-body problem in which the bodies rotate at the vertices of a regular tetrahedron, and of the 5-body problem where the bodies are at the vertices of a uniformly rotating pentatope (4-simplex) in $\mathbb{S}^{3}$. All solutions described so far are positive elliptic-elliptic RE.

For negative curvature, unusual orbits occur too. For instance, certain negative hyperbolic RE of the 3-body problem move in $\mathbb{H}^{3}$ like airplanes flying in formation, all on the same moving geodesic: the pilot in the middle sees always the other two planes left and right of him, as if unmoved. Such orbits are not even quasiperiodic, and don't come close to any periodicity at all.

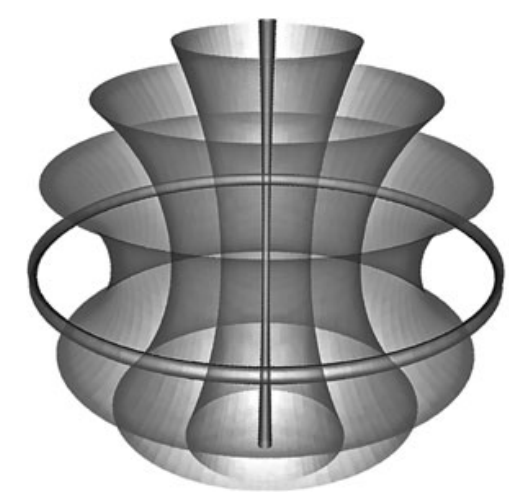

Figure 4. A 3-dimensional projection of a 4-dimensional foliation of the sphere $\mathbb{S}^{3}$ into Clifford tori.

The RE of $\mathbb{S}^{3}$ live on Clifford tori, which are tori of $\mathbb{R}^{4}$ :

$$
\mathbf{T}_{r \rho}^{2}=\left\{(w, x, y, z) \mid r^{2}+\rho^{2}=1,0 \leq \theta, \phi<2 \pi\right\},
$$

where $w=r \cos \theta, x=r \sin \theta, y=\rho \cos \phi$, and $z=\rho \sin \phi$. Unlike the standard torus of $\mathbb{R}^{3}$, these surfaces are flat. Moreover, they are subsets of $\mathbb{S}^{3}$. Each Clifford torus divides $\mathbb{S}^{3}$ into two solid tori, forming the boundary between them. The sphere $\mathbb{S}^{3}$ can also be foliated into Clifford tori, as Figure 4 shows. The RE of $\mathbb{H}^{3}$ live instead on hyperbolic cylinders,

$$
\mathbf{C}_{r \eta}^{2}=\left\{(w, x, y, z) \mid r^{2}-\eta^{2}=-1,0 \leq \theta<2 \pi, \xi \in \mathbb{R}\right\},
$$

where $w=r \cos \theta, x=r \sin \theta, y=\eta \sinh \xi, z=\eta \cosh \xi$. Unlike the Clifford tori, they are surfaces of positive Gaussian curvature. As in the case of the RE of $\mathbb{S}^{3}$, the qualitative behaviour of each type of orbit in $\mathbb{H}^{3}$ can be described, but we are not going into these details here.

Instead, I briefly discuss some simple orbits, which bear the name of Lagrange, who discovered them in 1772 in the Euclidean case. These RE are formed by equilateral triangles that rotate around the centre of mass. In $\mathbb{R}^{2}$ the masses can have any values, but in $\mathbb{S}^{2}$ and $\mathbb{H}^{2}$ they must be equal, a property that occurs because spheres and hyperbolic spheres have fewer symmetries than flat space.

To understand the importance of this remark, let us recall an old problem Gauss dealt with: the shape of physical space. In the early 1820s, he went as far as to measure the angles of the triangle formed by three mountain peaks near Göttingen (Inselberg, Brocken, and Hoher Hagen), to see whether their sum deviated from $\pi$ radians $([7,8,21,30])$ and thus prove space hyperbolic or elliptic. His experiment failed because he found no deviation beyond the inevitable measurement errors. Gauss's method cannot provide an answer for cosmic triangles because we cannot reach distant stars to measure the required angles. But if we can mathematically prove the existence of celestial orbits specific only to one of the hyperbolic, flat, or elliptic space, and none of the other two, then we might be able to decide on the shape of physical space by seeking such orbits in the night sky through astronomical observations. We know, however, that Lagrangian orbits exist in our solar system, as for example those formed by the Sun, 
Jupiter, and any of the Greek or Trojan asteroids. In the light of the previous result on Lagrangian orbits, does this mean that, at least for distances of order of $10 \mathrm{AU}$, space is Euclidean?

Such a conclusion would be premature, but we might not be too far from it. We would first have to make sure that the equality of the masses must also occur in $\mathbb{S}^{3}$ and $\mathbb{H}^{3}$, and that there are no quasiperiodic orbits of nonequal masses in curved space that are close to the Lagrangian ones. We did not prove such results yet, and don't even know whether they are true. But this is an incentive to better understand the equations of the curved and flat $N$ body problem.

\section{Rotopulsators}

An interesting class of orbits in the Euclidean case is that of homographic solutions. As their name suggests, they form configurations that are self-similar during the motion. But on spheres and hyperbolic spheres the only similar configurations are the congruent ones, since angles change when increasing or decreasing a given shape, even if the distances remain proportional. Of course, if viewed in the background space, the Euclidean figures are similar, but we like to think in terms of the space's natural geometry.

I therefore needed a new name for orbits given by configurations that rotate and expand or shrink at the same time. After some reflection, I came up with the term rotopulsator, which captures the above properties without implying the geometrical similarity of the configuration. We can think of an orbit that, say, only expands, as having a single pulse. The definitions were easy to obtain from RE, by allowing dilation and contraction and, therefore, a nonconstant angular velocity. So I was led to 5 classes of rotopulsators: 2 in $\mathbb{S}^{3}$ and 3 in $\mathbb{H}^{3}$, and I started studying them with my students Shima Kordlou and Brendan Thorn $[10,17]$. Then I also employed a young man, Sergiu Popa, who-due to personal circumstances-is currently not enrolled in the academic system, but has high talent for mathematics and does excellent research. We started developing this topic and found many new classes of orbits.

But instead of describing such solutions, I will reveal a trap I fell into, which shows why research in uncharted territory must be advanced with care and restraint. In the class of what I called positive elliptic-elliptic rotopulsators (orbits with two rotations in $\mathbb{S}^{3}$ ), I checked to see whether Lagrangian orbits of equal masses occur in the 3-body case. They do, but the ones I found were very strange: the sides of the equilateral triangle are constant, so there is no expansion or contraction. In other words, they are RE. Still, there is no element in the natural underlying subgroup $S O(2) \times S O(2)$ of $S O(4)$ that can generate this orbit. This orbit, however, passes at every instant through a continuum of $S O(2) \times S O(2)$ elements.

Since researchers in geometric mechanics define RE as orbits for which there is an element of some Lie group that can generate the orbit, I had apparently stumbled into an example that showed their definition to be inadequate. And for a couple of weeks I thought that this was the case, so I told my friends Tudor Ratiu and James Montaldi about it. In the end, they gave me a new angle from which to look at the problem, and soon I understood that I had been wrong.

There is a classical result in Lie group theory (of which I was not aware before), which says that every element of a semisimple compact Lie group lies in some maximal torus. $S O(4)$ is such a Lie group, and its maximal tori are of the form $S O(2) \times S O(2)$. By fixing a coordinate system in $\mathbb{R}^{4}$, we also define two rotations, so we fix the underlying $S O(2) \times S O(2)$ subgroup. And, indeed, in this naturally fixed subgroup, there is no element that could generate the Lagrangian RE. But there is always another system of coordinates, which fixes a different torus, where we can find such an element, as the theorem taught me. In other words, I had looked at the Lagrangian $\mathrm{RE}$ in the wrong coordinate system. It was like watching the uniform motion of a point around a circle in the projection of this circle (an ellipse) on some inclined plane. Pursuing the due diligence helped me evade the trap. It was not the first time. Traps, in various disguises, showed up and will likely show up again in this world where many things are surprising and unexpected.

\section{Stability}

Trying to decide the curvature of physical space using the idea described above would make little sense if the orbits found mathematically to exist are not stable. Of course, the stability of an orbit does not guarantee that it occurs in nature, but instability makes certain that we won't find it in the universe. So studying the stability of orbits is an important, though difficult, mathematical task.

There are many kinds of stability for solutions of ordinary differential equations. The most desirable is the one defined by Lyapunov, which assures that nearby orbits stay close for all time. But this property is rare, and the more so in celestial mechanics, where what one usually hopes for is orbital stability, which means that at least the orbits, but not necessarily the positions and velocities of the bodies, remain close to each other when small perturbations occur. Most of the time, however, we are content to prove even weaker properties, such as linear stability, which does not necessarily imply stability in nature, although it gives hopes for it.

In Euclidean space we know that the Lagrangian orbits are Lyapunov stable if one of the masses is very small, but unstable if the masses are comparable. Therefore one would expect that these orbits are unstable in curved space, since they occur only for equal masses. But this conclusion might not even be true. In a recent paper, Regina Martínez and Carles Simó showed that there are zones of linear stability for the Lagrangian orbits on $\mathbb{S}^{2}$ [29]. As expected, that does not happen when the equilateral triangle is small, since we get close to the Euclidean case, but it occurs when the size of the system is large enough. Similar things happen for other types of orbits, such as the tetrahedral ones in the 4-body problem in $\mathbb{S}^{2}$, as shown in [11].

\section{Perspectives}

When I present this topic to seasoned researchers, they often ask me whether I tried to answer this or that question about the equations of motion. Most of the time my answer 
is no. There is so much to discover in this new world (and the questions come naturally, by comparing what was done in the Euclidean case, which has been researched for more than 3 centuries) that, with my few collaborators and students, I have only scratched the surface so far.

This problem also lays bridges between several branches of mathematics: differential equations, dynamical systems, geometric mechanics, non-Euclidean and differential geometry, the theory of polytopes, geometric topology, Lie groups and algebras, and stability theory [11], while also showing potential for applications.

Obvious mathematical questions to ask are related to regularizing collisions and better understanding the other singularities that show up; discovering more non-equal mass orbits and determining their stability (spectral, linear, orbital, nonlinear); finding the bifurcations that occur when passing from negative to positive curvature through the Euclidean case, developing a 3D theory in terms of intrinsic equations, which were written only in 2D so far; seeking new RE and rotopulsators and understanding their properties; solving the curved version of Saari's conjecture and the corresponding Smale problem, which seems much harder than in the Euclidean case; and understanding whether chaotic behaviour shows up. Approaching these questions will generate other questions, as happens in every branch of mathematics.

A recent attempt also shows the way into the field of partial differential equations: I started working with my colleague Slim Ibrahim and our student Crystal Lind on understanding the Vlasov-Poisson equations for stellar dynamics in spaces of constant curvature, which provide the natural extension of the curved $N$-body problem to kinetic theory. This question is independent of everything mentioned in the previous paragraph and it will likely have a life of its own.

I have illustrated here both the risks and the rewards encountered when stepping into a new world. Thoughts of this kind belong to our research lives, but they never make it into journal papers. I have also tried to convey the idea that mathematics is not, as many people think, just about proving a famous conjecture, but it is first of all about finding new viable paths that keep our curiosity alive. The great masters knew that too.

\section{REFERENCES}

[1] R. Abraham and J. E. Marsden. Foundations of Mechanics (2nd ed.) Benjamin-Cummings, Reading, MA, 1978.

[2] J. Bertrand, Théorème relatif au mouvement d'un point attiré vers un center fixe, C. R. Acad. Sci. 77 (1873), 849-853.

[3] J. F. Cariñena, M. F. Rañada, and M. Santander, Central potentials on spaces of constant curvature: The Kepler problem on the two-dimensional sphere $\mathbb{S}^{2}$ and the hyperbolic plane $\mathbb{H}^{2}, \mathrm{~J}$. Math. Phys. 46 (2005), 052702.

[4] W. Bolyai and J. Bolyai, Geometrische Untersuchungen, Hrsg. P. Stäckel, Teubner, Leipzig-Berlin, 1913.

[5] F. Diacu, On the singularities of the curved $N$-body problem, Trans. Amer. Math. Soc. 363, 4 (2011), 2249-2264.
[6] F. Diacu, Polygonal homographic orbits of the curved 3-body problem, Trans. Amer. Math. Soc. 364 (2012), 2783-2802.

[7] F. Diacu, Relative equilibria of the 3-dimensional curved $n$-body problem, Memoirs Amer. Math. Soc. (to appear).

[8] F. Diacu, Relative equilibria of the curved N-body problem, Atlantis Press, Paris, 2012.

[9] F. Diacu, T. Fujiwara, E. Pérez-Chavela, and M. Santoprete, Saari's homographic conjecture of the 3-body problem, Trans. Amer. Math. Soc. 360, 12 (2008), 6447-6473.

[10] F. Diacu and S. Kordlou, Rotopulsators of the curved N-body problem, arXiv:1210.4947.

[11] F. Diacu, R. Martínez, E. Pérez-Chavela, and C. Simó, On the stability of tetrahedral relative equilibria in the positively curved 4-body problem, Physica D (to appear).

[12] F. Diacu and E. Pérez-Chavela, Homographic solutions of the curved 3-body problem, J. Differential Equations 250 (2011), 340-366.

[13] F. Diacu, E. Pérez-Chavela, and M. Santoprete, Saari's conjecture for the collinear N-body problem, Trans. Amer. Math. Soc. 357, 10 (2005), 4215-4223.

[14] F. Diacu, E. Pérez-Chavela, and M. Santoprete, The N-body problem in spaces of constant curvature. Part I: Relative equilibria, J. Nonlinear Sci. 22, 2 (2012), 247-266.

[15] F. Diacu, E. Pérez-Chavela, and M. Santoprete, The N-body problem in spaces of constant curvature. Part II: Singularities, J. Nonlinear Sci. 22, 2 (2012), 267-275.

[16] F. Diacu, E. Pérez-Chavela, and J. Guadalupe Reyes Victoria, An intrinsic approach in the curved $\mathrm{N}$-body problem. The negative curvature case, J. Differential Equations 252 (2012), 4529-4562.

[17] F. Diacu and B. Thorn, Rectangular orbits of the curved 4-body problem, arXiv:1302.5352.

[18] A. Einstein, L. Infeld, and B. Hoffmann, The gravitational equations and the problem of motion, Ann. of Math. 39, 1 (1938), 65-100.

[19] V. A. Fock, Sur le mouvement des masses finie d'après la théorie de gravitation einsteinienne, J. Phys. Acad. Sci. USSR 1 (1939), 81-116.

[20] I.M. Gelfand and S.V. Fomin, Calculus of Variations, PrenticeHall, Englewood Cliffs, NJ, 1963.

[21] G. Goe, Comments on Miller's "The myth of Gauss's experiment on the Euclidean nature of physical space," Isis 65, 1 (1974), 83-87.

[22] W. Killing, Die Rechnung in den nichteuklidischen Raumformen, J. Reine Angew. Math. 89 (1880), 265-287.

[23] V. V. Kozlov and A. O. Harin, Kepler's problem in constant curvature spaces, Celestial Mech. Dynam. Astronom 54 (1992), 393-399.

[24] T. Levi-Civita, The relativistic problem of several bodies, Amer. J. Math. 59, 1 (1937), 9-22.

[25] H. Liebmann, Die Kegelschnitte und die Planetenbewegung im nichteuklidischen Raum, Berichte Königl. Sächsischen Gesell. Wiss., Math. Phys. Klasse 54 (1902), 393-423.

[26] H. Liebmann, Über die Zentralbewegung in der nichteuklidische Geometrie, Berichte Königl. Sächsischen Gesell. Wiss., Math. Phys. Klasse 55 (1903), 146-153.

[27] R. Lipschitz, Untersuchung eines Problems der Variationsrechnung, in welchem das Problem der Mechanik enthalten ist, $J$. Reine Angew. Math. 74 (1872), 116-149. 
[28] N. I. Lobachevsky, The new foundations of geometry with full theory of parallels [in Russian], 1835-1838, In Collected Works, V. 2, GITTL, Moscow, 1949, p. 159.

[29] R. Martínez and C. Simó, On the stability of the Lagrangian homographic solutions in a curved three-body problem on $\mathbb{S}^{2}$, Discrete Contin. Dyn. Syst. Ser. A 33 (2013), 1157-1175.

[30] A. I. Miller, The myth of Gauss's experiment on the Euclidean nature of physical space, Isis 63, 3 (1972), 345-348.

[31] E. Pérez-Chavela and J.G. Reyes Victoria, An intrinsic approach in the curved $\mathrm{N}$-body problem. The positive curvature case, Trans. Amer. Math. Soc. 364, 7 (2012), 3805-3827.
[32] D. Saari, Collisions, Rings, and Other Newtonian N-body Problems, American Mathematical Society, Regional Conference Series in Mathematics, No. 104, Providence, Rl, 2005.

[33] E. Schering, Die Schwerkraft im Gaussischen Raume, Nachr. Königl. Gesell. Wiss. Göttingen 13 July, 15 (1870), 311-321.

[34] A.V. Shchepetilov, Nonintegrability of the two-body problem in constant curvature spaces, J. Phys. A: Math. Gen. V. 39 (2006), 5787-5806; corrected version at math.DS/0601382.

[35] S. Smale, Mathematical problems for the next century, Mathematics: frontiers and perspectives, Amer. Math. Society, Providence, RI, 1999, pp. 271-294. 